GABRIELA MEDRANO

Profesora asistente

Escuela de Arquitectura, Universidad San Sebastián

Santiago, Chile

\section{FELIPE ZEGERS}

Colectivo Blá!

Santiago, Chile



Adopted by the UN towards the end of 1948, after the atrocities committed in World War II, the Universal Declaration of Human Rights is perhaps the most important document ever written, and one of the texts that has been translated into more languages. By protecting the rights inherent to our human condition, this is a text that we should all know and respect. "Inclusive information" seeks precisely that: to expand access to knowledge of those minimum rights, which constitute us as humans.
1 Información

inclusiva 01, Museo de la Memoria y los Derechos Humanos, Quinta Normal, mayo de 2019. / Inclusive Information 01, Museum of Memory and Human Rights, Quinta Normal, May, 2019.

(c) M. Fernanda Landin.

\section{Keywords}

Laws

Urban intervention

Language

Human Rights

Portfolio 




2 Información inclusiva 02, Museo de la Memoria y los Derechos Humanos, Quinta Normal, mayo de 2019. / Inclusive Information 02, Museum of Memory and Human Rights, Quinta Normal, May, 2019.

(c) M. Fernanda Landin. 

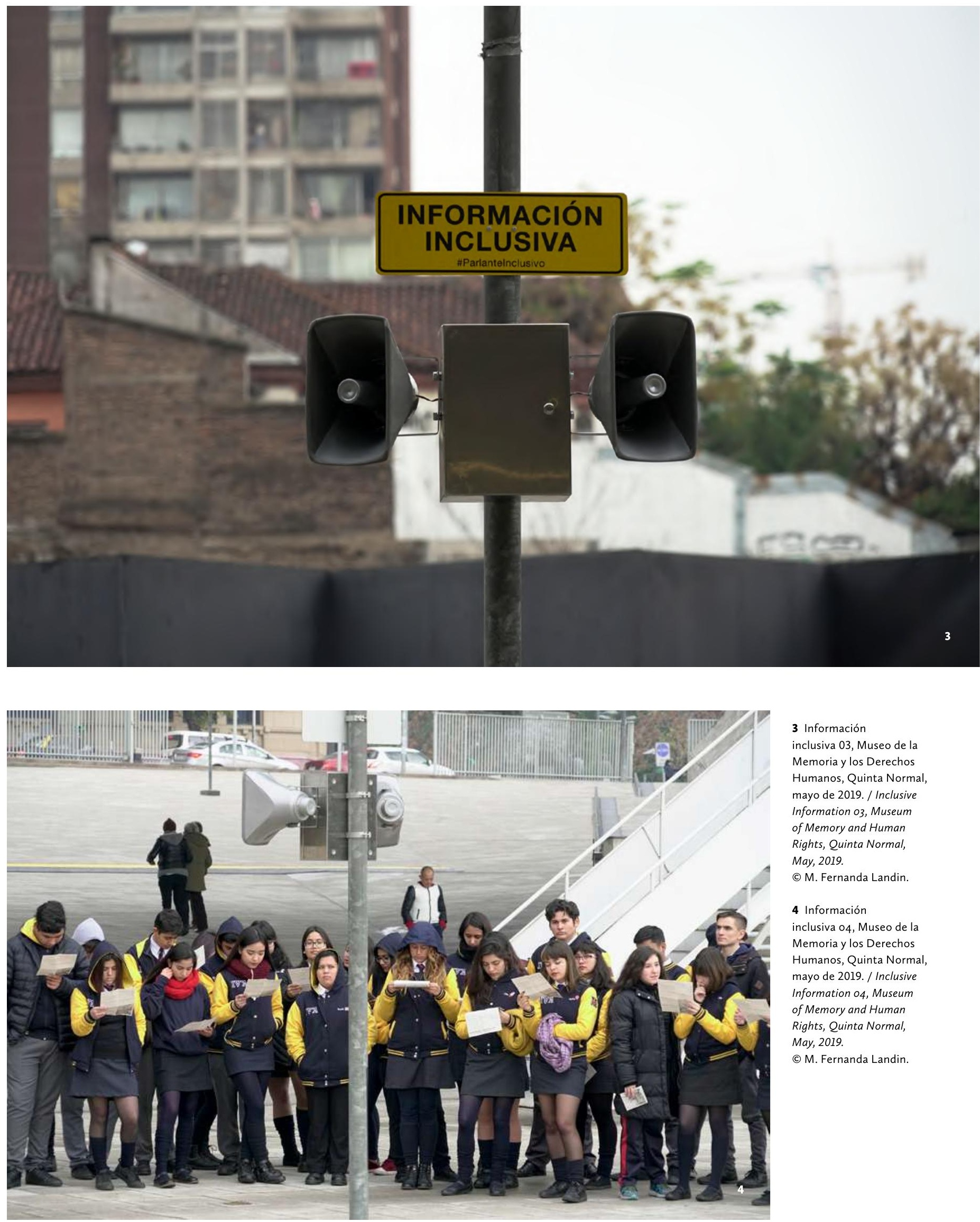

3 Información inclusiva 03 , Museo de la Memoria y los Derechos Humanos, Quinta Normal, mayo de 2019. / Inclusive Information 03, Museum of Memory and Human Rights, Quinta Normal, May, 2019.

(c) M. Fernanda Landin.

\section{Información}

inclusiva 04, Museo de la Memoria y los Derechos Humanos, Quinta Normal, mayo de 2019. / Inclusive Information 04, Museum of Memory and Human Rights, Quinta Normal, May, 2019.

(c) M. Fernanda Landin. 


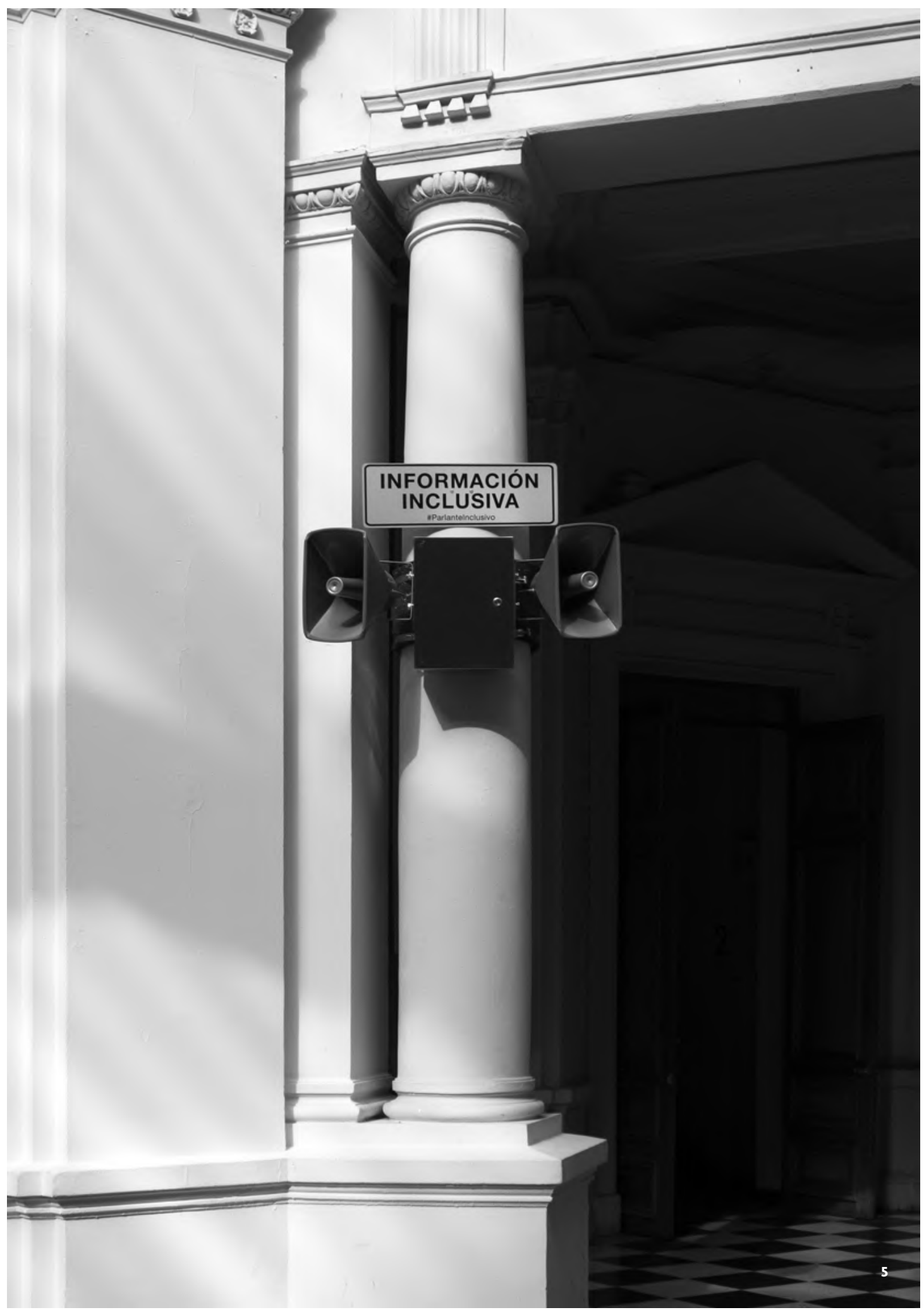

5 Información inclusiva 05, Museo de Arte Contemporáneo, Santiago, octubre de 2019. / Inclusive Information 05, Museum of Contemporary Art, 


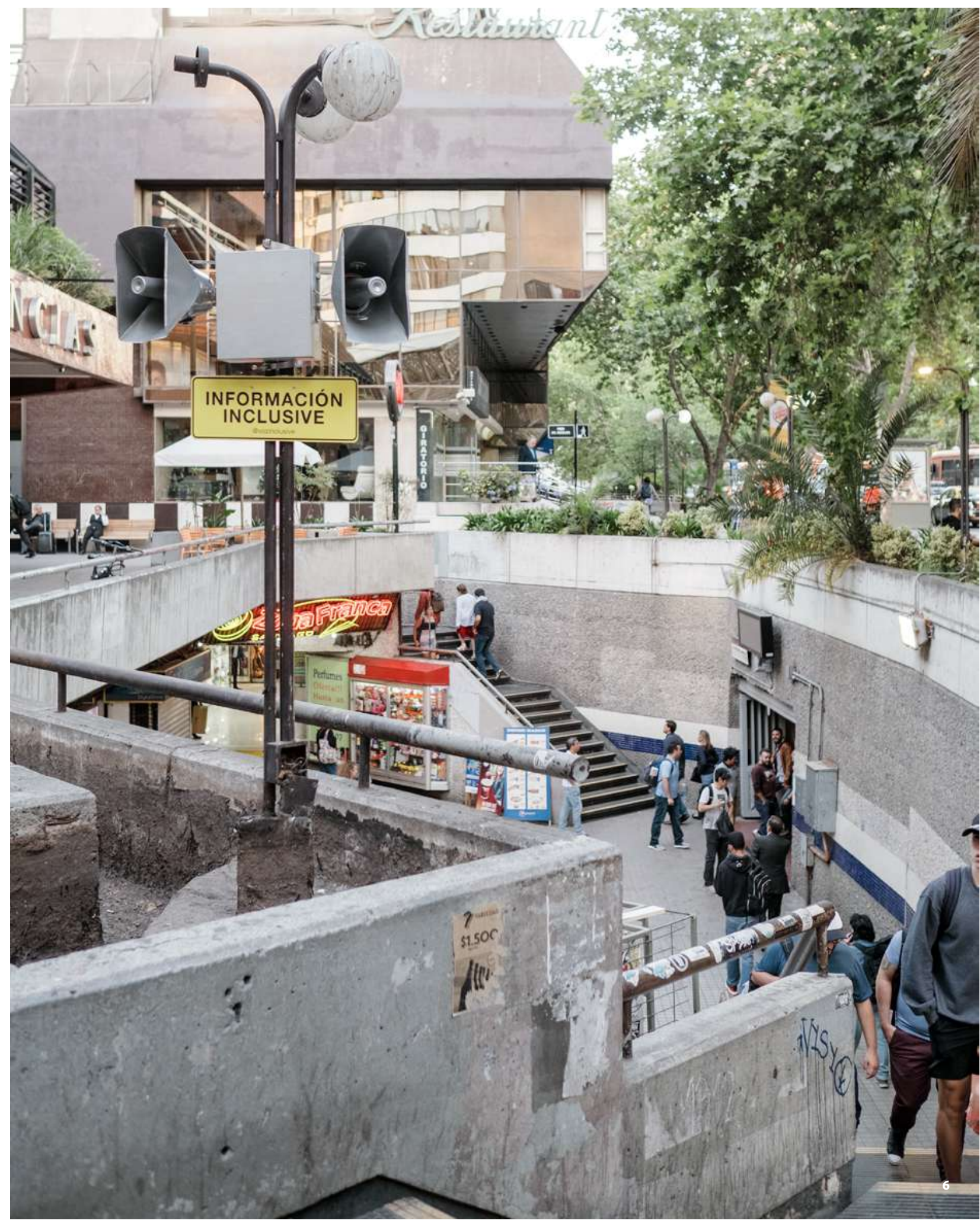




\section{LE DECLARACIÓN UNIVERSAL DE LES Dereches Humanes}

CONSIDERANDO que le libertad, le justicie y le paz en el mundo tienen por base el reconocimiento de le dignidad intrínseca y de les dereches iguales e inalienables de todes les miembres de le familie humane; CONSIDERANDO que el desconocimiento y el menosprecio de les dereches humanes han originado actos de barbarie ultrajantes para le conciencie de le humanidad, y que se ha proclamado, como la aspiración más elevada de le persone, el advenimiento de un mundo en que les seres humanes, liberados del temor y de la miseria, disfruten de le libertad de palabra y le libertad de creencias;

CONSIDERANDO esencial que les dereches humanes sean protegidos por un régimen de Dereche, a fin de que le persone no se vea compelido al supremo recurso de le rebelión contra la tiranía y la opresión;

CONSIDERANDO también esencial promover el desarrollo de relaciones amistosas entre les naciones;

CONSIDERANDO que les puebles de les Naciones Unides han reafirmado en le Carte su fe en les dereches fundamentales de le persone, en la dignidad y el valor de le persone humane y en le igualdad de les dereches de les persones, y se han declarado resueltos a promover el progreso social y a elevar el nivel de vida dentro de un concepto más amplio de le libertad; CONSIDERINDO que les Estades Miembres se han comprometido a asegurar, en cooperación con le Organización de les Naciones Unides, el respeto universal y efectivo a les dereches y les libertades fundamentales del ser humane;

CONSIDEMNDO que una concepción común de estos dereches y libertades es de la mayor importancia para el pleno cumplimiento de dicho compromiso; POR LO TANTO LE ASAMBLEE GENERAL DE LES NACIONES UNIDES proclama Le Declaración Universal De Les Dereches Humanes como ideal común por el que todes les puebles y les naciones deben esforzarse, a fin de que tanto les individues como les instituciones, promuevan, mediante la enseñanza y la educación, el respeto a estos dereches y libertades, y aseguren, por medidas progresivas de carácter nacional e internacional, su reconocimiento y aplicación universales y efectivos, tanto entre les puebles de les Estades Miembres como entre los territorios colocados bajo su jurisdicción.

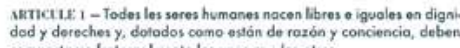

sRTici102-1. Todo persone fiene les dereches y les bibertodes procle. modos en lo presento Declotoción, un distinción olgúne de raza, coler. Iexe, idiome, teligión, opinión politique o de cualquier otra indolo.

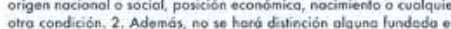
la condición polîique, juridique o internacionel da lo pais o territor"s de cuya jurisdicción dependo une persone, tanto si se itrata de un pai indopendiente, como de un territorio bojo odministroción fiduciorio no outónnomo a sometido a cualquier otra limitación do soberenia.

MrticuLo 3 - Tode individue tiene dereche o le vide, a le libertad a lo seguridod do se pervone bumone.

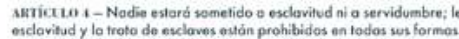
ArriciLos - Ningún individue sero sometido a torturas ni a penas o trotos crueles, inhumines o degredantes

arrictio 6 - Tode ser humane fiene dereche, en todas portes, a reconocimiento de su personalidod jeridique

akricule 7 - Todes son iguales onte le loy y tienen, sin distincíbe dereche a iguol prolección de le lay. Todos tienen dereche a igual
protección contra toda discriminación que intrinja esta Decloroción y protacción contra toda discriminación que intion.
contro toda provocación o tal discriminación.

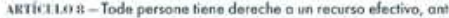
les tribunalos nocionoles competontes, que le ampore contro octos que
violon sus dereches fundamentales reconocidor por lo constitución o violon sus
por le ley,

skTiciLo 9 - Ningune persone podra ser orbitratiomente detenido AlTicino $9-\mathrm{Ning}$
proso ni desterroda.

sRrictio 10 - Tode persone tiene dereche, en condiciones de pleno igvoldod, a ver oido públicamente y con jufficio por un tribunal inde.

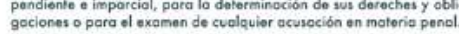

мктіст10 11 - 1. Tode persone ocusada de delito tiene dereche que se presuma su inocencia mientras no se pruebe su culpobilidad, conforme a le ley y en juicio público en al que se lo hoyan asegurodo

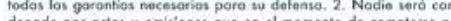
fueron delictives segin lo Dereche nacional o internocional. Tamporse

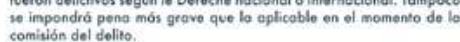

skrictu 12 - Ningune persono será objeto de injerencias arbitrarias en su vide privado, su familie, su domiclitio o su correspandencia, n

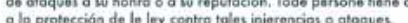

AnTictio is - 1. Tode porsone tiene doreche o circulor libremente

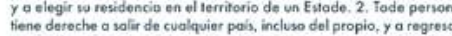
a su pois.

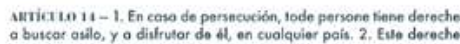

no podrá ser invecodo contra una occión ivdicial reolmente originoda por delías corrunes o por actos opuestos a los propésitos y principion AltrictLo 15 - 1, Tode persone tiene dereche o une nocionalidod. 2 A ningune persone so le privoró crbitrariomente de wa nocionolidod ni del coreche a cambiar do nocionalided.

Atrictuo $16-1$, Todes les sares humones a partir do la eded núbil, dadonan dereche, Lin routricciba olguno por motives de roza, nacionalidereches en cuanto a lo matrimonie, dorante le matrimonie y en caso de dirolución de le matrimonie. 2. Sólo mediante libre y pleno conto fanifio es el elemento natural y fundamentol de le sociodod y tiene dereche a la protocción do lo sociedod y do lo Estade.

Atricu Lo 1:-1. Tode persone fiene dereche a lo propiedod, individuol y colectivanente. 2 Ningune parsone seré privode arbàrariomente
de su propiedod. Skria Lo is - Tode persone tiene dereche a le libertod de pens: de combiar de religión o de craencis a cic como lo liberted de maniestar su ret gión o su creencio, individual y colectivamente, tonto mn público como en privodoo, por la enseñanzo, la próctica, el culo y lo observoncia.

งkric 150 19 - Tode individue tiene dereche a le libertod de opinión y do expresión; osto derecho incluye no ser molestado a causo de

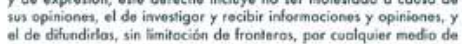
expresión

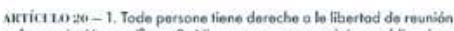
y de asocioción pacificas. 2. Ningune persone podrá ser obligoda a

Artia L 21 - 1. Tode persone tiene dereche o porticipor en le gobiorne de w pois, directamente o por modio de represientanter libremente escogides. 2. Todo persone fiene derecha de acceso, en condiciones de igualdad, a les huncioness públicas do su pais. 3 . La voluntad do lo

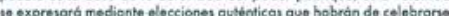
peribidicamente, por sufrogio univertsol a igual y por voto 10 creto o otro procedimiento equivalenta que goronfice la thertod del voto.

skric 10 xa - Todo persone, como miembro de lo sociedod, tiene Seroche a la seguridad tocial, y o obiener, modiante ol asfuerzo zoción y los recursos de cado Eutode, la satíf́acción de les dereches econdinicos, tociolos y culturoles, inditipensobles a su dignidad y ol
libre desorrollo de w personalidod.

Akriaio za - 1. Tode persone tiene dereche a la libre elocción de su trabojo, a condiciones equitatives y lakiffoctorios da trabojo y o la proteccion contra el desersplea. 2. Tade persone tiene dereche. persone que traboja tiene dereche a una remunereción equitativa y solisfactorio, que lo asegure, asi como a su fomilio, uno existencia conforme a la dignidad humane, $y$ que seró completada, an caso neccesorio, por cualesquiera otros modios de protección social. 4. rodo parsono tiene doreche a tundar sindicotos y a sindicerse pora Auriatio 21 - Tode pernone fiene dereche al descanio, el distute
del tiempo libro, a una linitación rozonoble do lo duración del trobojo y a vocociones peribdicas pogados

AlTictio $2 s-1$. Tade perrone tiene dereche o un nivel de vido ade. cuodo quen lo asegure, ani como a su fannilio, la salud y el bienastar,

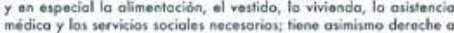
las seguros en coso de desempleo, enformedod, involidez, viudez. vejez y otros cosos do peredida do sus modios de wubsistencia por infancie tienen dereche o cuidodos $y$ asistencis especicles. Todes los hifies tienten dereche a igual protección roded.

งหTicite $26-1$. Tode persone tiene derecte a le edvcoción. te edv cación dobe ser protuâta, al menos en lo concernionte a la instrucción olemantal y fundamontal. La instrucción olemental serd́ obligotoria. occesso a los estudios superiones sorá igual para todes, en función de los méritos respectivos. 2. Le educación tendrá por objeto el pleno a les dereches humares y a los libertodes fundorentoles for rospecere lo comprensión, la toleroncia $y$ la amistod ontre todes les nociones y todes les grupes etreices o roligiososy y promoverá el desorrollo do pQz. 3. Les padres teodrán deracho proterente a escoger al tipo da

utriciLe $2 z-1$, Tode persone tinae derache a tomor parte libremete on la vido cultural de le comunidad, o gozer de las artes y a porticipor persona tieno derecheo o la protección de los intereses morales y mote riales que lo correspondon por razón de los producciones cientificas. literories o artiticices de que sea autor.

ARTíato zs - Todo persone fiene dereche a que se estoblezca un orden social e internocional en el que les dereches y los libertodes

Atrictito $29-1$. Tode persone tiene deberes respecto o le come nidad, puesto que soblo on le comvnidod puede descrroillor libre y

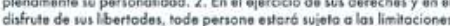
establecidas por le ley con el fin de aregurar el reconocimiento y el respeto do los dereches y les fibertodes do los demós, y de sosisfocet las justos oxigencios de la morol, del orden péblico y del bienestar generol en une sociedad democrtíque. 3. Elas doeseches $y$ libertodes no podrán en ningún coso ser ojarcidos en oposición a los propósito

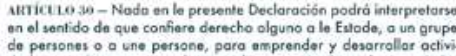
dodes o realizat octos tendientes a la supresión de cualquiero de le derechos y les libertades proclamados en esto Decloracion Acotscáx as - Tode persone tiene derecho a equivocarso.

scotscióx sz - Tode persone tiene derecho a cambior de opinión. scatscaiv as - Tode parsone tiene dereche a irso de donde une está.

\section{INFORMACIÓN INCLUSIVA}

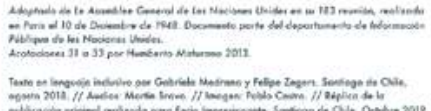


nclusive Information is a citizen intervention

- a temporary intervention in the public space

through artifacts or devices that use the urban infrastructure as support, modifying everyday life that provides an invitation to reflect on the use of language as a generator of reality.

Language establishes a close relationship with thought, as a means of communication, interpreting the reality in which we live, and reflecting what society is at all times. We propose a democratization of our language, which has, by default, fixed the masculine linguistic gender in most of the institutions that represent us as a society.

Inclusive Information is made up of a sound device - Inclusive Speaker - that uses the existing urban infrastructure as a framework to temporarily settle in public space and disseminate civic texts, in this case, the Universal Declaration of Human Rights, in inclusive language. This artifact works in two modes, the continuous sound reproduction of a female murmur - making it a participatory actor within the space - and a performance in which a complete public reading is made using the Replica print, a graphic reproduction of the Universal Declaration of Human Rights of 1948 but modified by means of inclusive language.

Our intervention has been installed in public spaces in the streets of Córdoba, Buenos Aires and Santiago, as well as in the Museo de la Memoria y de los Derechos Humanos, during the $x \mathrm{x}$ I Biennial of Architecture and Urbanism of Chile, and in the Museum of Contemporary Art, at a time when the museum was forced to close due to the October 18 uprising. Because of this, it kept on reproducing the Universal Declaration of Human Rights for 44 continuous hours. ARQ

\section{Gabriela Medrano}

<gmedrano.arq@gmail.com >

Architect and Master in Architecture, Universidad Andrés Bello, 2008. She has won several international architecture competitions, such as the Bío-Bío Regional Theater, in association to Smiljan Radic and Eduardo Castillo. She currently teaches at the Universidad Andrés Bello and at the Universidad San Sebastián. Since 2018, she has carried out citizen interventions. 